\title{
A splitting algorithm for a novel regularization of Perona-Malik and application to image restoration
}

\author{
Fahd Karami*, Lamia Ziad and Khadija Sadik
}

\begin{abstract}
In this paper, we focus on a numerical method of a problem called the Perona-Malik inequality which we use for image denoising. This model is obtained as the limit of the Perona-Malik model and the $p$-Laplacian operator with $p \rightarrow \infty$. In Atlas et al., (Nonlinear Anal. Real World Appl 18:57-68, 2014), the authors have proved the existence and uniqueness of the solution of the proposed model. However, in their work, they used the explicit numerical scheme for approximated problem which is strongly dependent to the parameter $p$. To overcome this, we use in this work an efficient algorithm which is a combination of the classical additive operator splitting and a nonlinear relaxation algorithm. At last, we have presented the experimental results in image filtering show, which demonstrate the efficiency and effectiveness of our algorithm and finally, we have compared it with the previous scheme presented in Atlas et al., (Nonlinear Anal. Real World Appl 18:57-68, 2014).
\end{abstract}

Keywords: Image restoration, Perona-Malik inequality, $p$-Laplacian, Splitting algorithm

\section{Introduction}

Image denoising is one of the fundamental challenges in the field of image processing and computer vision. The aim is to remove noise while preserving edges, boundaries, and textures. To handle this problem, partial differential equations [1-7], variational models [8-11], energy minimization, bilateral filtering [12, 13], and wavelet thresholding $[14,15]$ have been proposed depending on the domain of applications. Generally, the partial differential equations use a nonlinear anisotropic diffusion to restore a degraded image which they seek to improve its quality by removing noise while preserving details and even enhancing edges.

In 1990, Perona-Malik [2] proposed a nonlinear diffusion equation that succeeded in image denoising. Although, this model is an ill-posed problem in analytical point of view, besides, the numerical simulations produce the staircase effect. This paradoxical result has been named as the Perona-Malik Paradox [16]. Motivated by the ill-posedness of the Perona-Malik model, many

*Correspondence: fa.karami@uca.ma

Université Cadi Ayyad, Ecole Supérieure de Technologie d'Essaouira, B.P. 383 Essaouira El Jadida, Essaouira, Morocco works (see for instance [17-19]) suggest to introducing the regularization in space and/or time. Using the spatial convolution inside the anisotropic diffusion and replacing the diffusivity by a slight variation, new modified models have been proposed, but they produce an undesirable blurring effect. Otherwise, some new class of backwardforward regularizations has been introduced. In [20], the authors combine the Perona-Malik with a Laplacian operator and develop a new effective model, and they make a generalization of their results by replacing the Laplacian with a nonlinear $p$-Laplacian operator for $p \in(1,2]$ (cf. [21]). Recently, in [22], the authors proposed a new regularization based on the previous interpolation of PeronaMalik and $p$-Laplacian with large value of $p$. Their model is well posed, it reduces the staircase effect and avoids creating new features in the image, they have also done a study of the limiting problem. For a numerical purpose, the authors used an explicit finite difference scheme which is unstable and the condition of stability depends on the parameter $p$.

In this paper, we develop a numerical semi-implicit method approaching the Perona-Malik inequality by using 
a splitting method [23]. The idea is to decompose the limit problem into two operators:

- The Perona-Malik operator which will be treated by the additive operator schemes [24].

- The second is called the sub-differential flow, where we use the Euler implicit discretization as well as we develop a dual formulation associated to the minimization problem [25].

The rest of this paper is organized as follows. In the next Section 2, we provide reviews of some previous works that are Perona-Malik model, $p$-Laplacian equation and their interpolation. In Section 3, we present a new numerical scheme using an operator splitting algorithm that splits the Perona-Malik inequality in two sub-models treated by additive operator schemes and dual formulation, respectively. Finally, some numerical simulations are given to demonstrate the effectiveness of the proposed algorithm.

\section{Reviews of some previous works}

In this section, we recall some previous models. Let $T>0$ be a fixed time, $f$ the intensity of the noisy image, $u$ the desired clean image that was corrupted with the noise $n$ such that $f=u+n$, and let $\Omega$ be a bounded picture domain with smooth boundary $\Gamma$.

\subsection{Perona-Malik model}

The Perona-Malik model is a powerful model and widely used in image denoising. Hence, the idea behind this model is to improve the results obtained by the PDE heat and to change the equation by introducing the edge detection process (the diffusivity coefficient). Perona-Malik problem is obtained by solving the following anisotropic diffusion equation with a Neumann boundary condition:

$$
\begin{cases}u_{t}-\operatorname{div}(g(|\nabla u|) \nabla u)=0 & \text { in } Q:=(0, T) \times \Omega, \\ \nabla u . \vec{n}=0 & \text { in } \Sigma:=(0, T) \times \Gamma, \\ u(x, 0)=f & \text { in } \Omega .\end{cases}
$$

The diffusion function $g$ is an edge indicator function that controls the diffusion process by reducing the effect of diffusion near edges and behaves locally as the heat equation. Typical choices of $g$ are:

$$
g(t)=\frac{1}{1+\left(\frac{t}{\lambda}\right)^{2}} \quad \text { or } \quad g(t)=\exp \left(-\frac{t^{2}}{2 \lambda^{2}}\right),
$$

where $\lambda>1$ is a threshold parameter that determines the size of the gradients which will be preserved and $g$ is a decreasing function worth $\lambda$ where $|\nabla u|$ is close to 0 and tend to 0 for large $|\nabla u|$. Note that if $g(.) \equiv 1$, we recover the heat equation. For the diffusivity function $g$, it follows the flux function $\Phi(t)=\operatorname{tg}(t)$ which satisfies $\Phi^{\prime}(t) \geq 0$ for $|t| \leq \lambda$ and $\Phi^{\prime}(t) \geq 0$ for $|t| \leq \lambda$.

We observe from the Fig. 1b that the Perona-Malik model is a forward parabolic type for $|t| \geq \lambda$ and a backward parabolic type for $|t| \leq \lambda$. This model is an ill-posed problem from the mathematical point of view and produces an unwanted phenomena well known by staircase effect.

\subsection{The $p$-Laplacian equation}

To obtain the $p$-Laplacian equation, we merely replace the diffusion function $g$ of the Perona-Malik equation by $g(t)=t^{p-2}$. The $p$-Laplacian equation has been proposed and studied by many authors (see for instance [26]). It plays an important role in the modeling of many phenomena in different areas such as image processing [27], sandpile [28, 29], and fluid mechanic [30]. The evolutionary $p$-Laplacian equation can be written as:

$$
\left\{\begin{array}{lr}
u_{t}-\operatorname{div}\left(|\nabla u|^{p-2} \nabla u\right)=0 & \text { in } Q, \\
\nabla u . \vec{n}=0 & \text { in } \Sigma, \\
u(x, 0)=f & \text { in } \Omega .
\end{array}\right.
$$

We recall that the case $p=2$, the model corresponds to a well-known heat equation. For $p=1$, the equation is
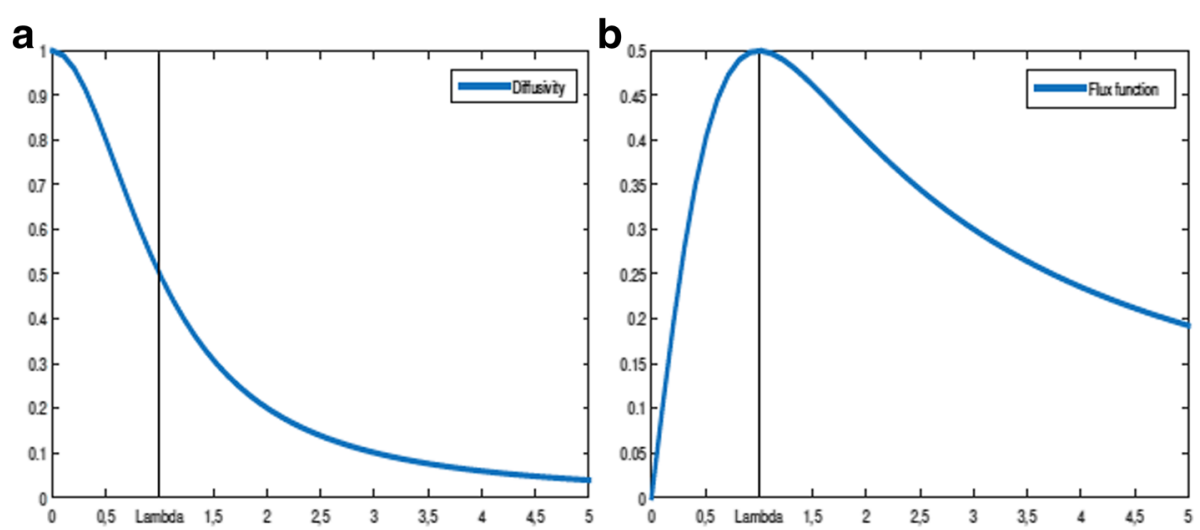

Fig. 1 a Left: diffusivity $g(t)$. b Right: flux function $\Phi(t)$ 

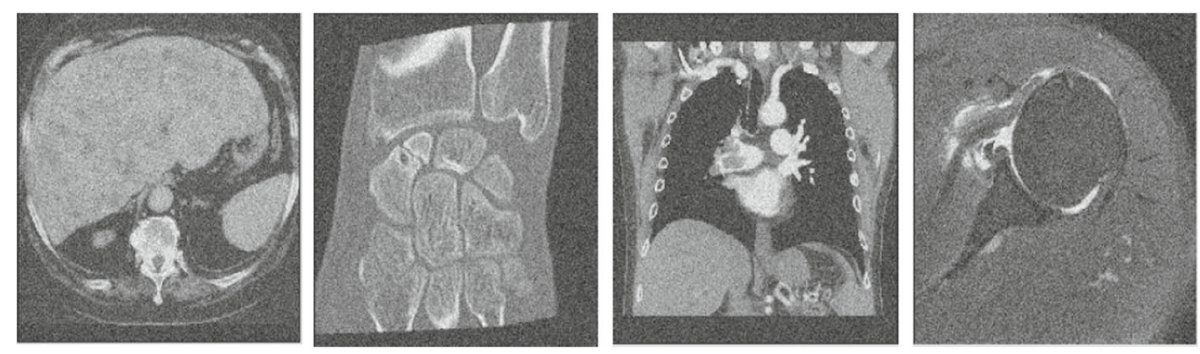

Fig. 2 Images corrupted by Gaussian noise with zero mean and variance $\sigma^{2}=0.025$

called total variation and for a large $p$, the model is known as the infinite Laplacian.

\subsection{Perona-Malik and $p$-Laplacian model}

The staircasing phenomena is a manifestation of the forward-backward nature of the Perona-malik equation which appears in its discretization. In order to escape the backward regime, a novel kind of regularization [20-22] of the classical Perona-Malik model is proposed for image processing. This regularization still allows gradient growth while controls its maximal size which is done by combining two classical models, Perona-Malik and $p$-Laplacian.

Indeed, the following regularization reduces the size of the backward region and the solutions can flee the backward region simply by developing small and large gradients:

$\left\{\begin{array}{lr}u_{t}-\operatorname{div}(g(|\nabla u|) \nabla u)-\frac{1}{\lambda^{p}} \operatorname{div}\left(|\nabla u|^{p-2} \nabla u\right)=0 & \text { in } Q, \\ \nabla u . \vec{n}=0 & \text { in } \Sigma, \\ u(x, 0)=f & \text { in } \Omega .\end{array}\right.$

This problem is well posed for a large $p$, and the existence and uniqueness of the asymptotic behavior as $p \rightarrow$ $\infty$ are established in [22]. Letting $p \rightarrow \infty$, the problem (1) converges to the following model:

$$
\left\{\begin{array}{lr}
u_{t}-\operatorname{div}(g(|\nabla u|) \nabla u)+\partial I_{K}(u) \ni 0 & \text { in } Q \\
\nabla u . \vec{n}=0 & \text { in } \Sigma, \\
u(x, 0)=f & \text { in } \Omega,
\end{array}\right.
$$

where

$$
K=\left\{\phi \in W^{1, r}(\Omega):|\nabla \phi| \leq \lambda \text { a.e. in } \Omega\right\},
$$

and $I_{K}($.$) denotes the indicator functional of the set K$, for all $u \in W^{1, r}(\Omega) I_{K}$ is defined by:

$$
I_{K}(u)=\left\{\begin{array}{lll}
0 & \text { if } & u \in K \\
+\infty & \text { if } & u \notin K
\end{array}\right.
$$

Thanks to Remark 3.2 of [22], for $p>\lambda^{2}+1$, the problem (1) has a unique weak solution $u_{p}$ and

$$
\begin{aligned}
& \text { for } p \rightarrow \infty, u_{p} \rightarrow u \text { weakly in } L^{r}\left(0, T, W^{1, r}(\Omega)\right) \\
& \text { for all } r>1 .
\end{aligned}
$$

Moreover, $u \in \mathcal{W} \cap \mathcal{K}^{t}$ is the unique solution of (2) in the following sense:

$$
\int_{0}^{T}\left\langle\frac{\partial u}{\partial t}, u-\phi\right\rangle d t+\int_{0}^{T} \int_{\Omega} g(|\nabla u|) \nabla u \nabla(u-\phi) \leq 0,
$$

for any $\phi \in \mathcal{K}^{t}=\left\{\xi \in L^{r}\left(0, T ; W^{1, r}(\Omega)\right): \xi(t) \in K\right\}$, having

$$
\begin{aligned}
\mathcal{W}= & \left\{\phi \in L^{r}\left(0, T ; W^{1, r}(\Omega)\right): \frac{\partial \phi}{\partial t}\right. \\
& \left.\in L^{r^{\prime}}\left(0, T ;\left(W^{1, r}(\Omega)\right)^{\prime}\right)\right\}, r>1 .
\end{aligned}
$$
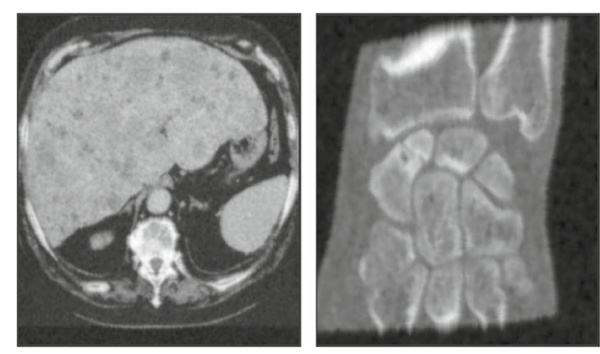
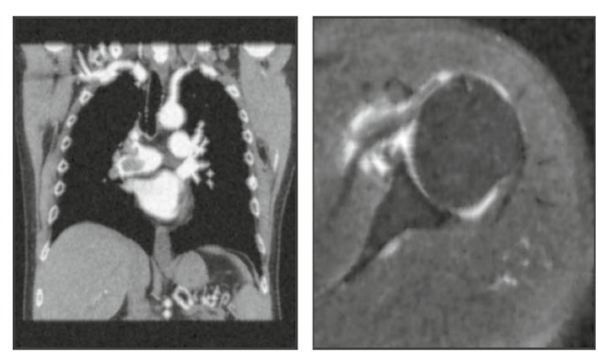

Fig. 3 Restored images by using our method 


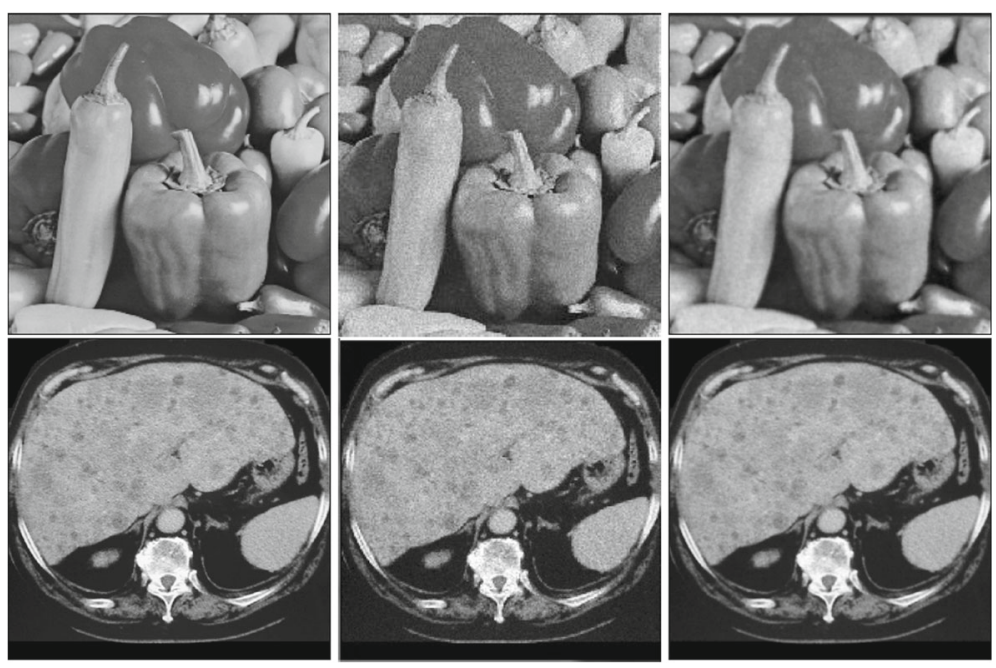

Fig. 4 Left column, original images; middle column, noisy images corrupted by poissonian noise; right column restored images with $\lambda=60$

For further details and another sophisticated notion of solutions, we refer to [22]. We remark that the limiting problem can be rewritten as:

$$
\left\{\begin{array}{l}
u_{t}-\operatorname{div}(g(|\nabla u|) \nabla u)-f \leq 0, \quad|\nabla u| \leq \lambda, \\
\left(u_{t}-\operatorname{div}(g(|\nabla u|) \nabla u)-f\right)(|\nabla u|-\lambda)=0,
\end{array}\right.
$$

which is equivalent to solving the Perona-Malik problem in the region where the norm of the gradient is less than $\lambda$.

\section{An operator splitting algorithm}

The operator splitting methods are very useful to derive fast algorithms. It is worthwhile to be used when the problem we want to solve has an additive structure, the main idea is to split the problem into sub-problems that are easier to solve by treating its summands separately in each iteration of the algorithm. To achieve this goal, we use of the operator splitting algorithm that splits the proposed model into two equations. The first one corresponds to solve the following Perona-Malik model:

$$
\begin{cases}u_{t}-\operatorname{div}(g(|\nabla u|) \nabla u)=0 & \text { in } Q, \\ \nabla u \cdot \vec{n}=0 & \text { in } \Sigma, \\ u(x, 0)=f & \text { in } \Omega .\end{cases}
$$

The second is a diffusion equation associated to the infinity Laplacian that is given by:

$$
\begin{cases}u_{t}+\partial I_{K}(u) \ni 0 & \text { in } Q \\ \nabla u . \vec{n}=0 & \text { in } \Sigma, \\ u(x, 0)=f & \text { in } \Omega .\end{cases}
$$

Let $N>0$ be given, $\tau=T / N$ be the time step, $t^{n}=n \tau, n=0, \ldots, N$, and let us consider $u^{n}$ an approximation of $u\left(t^{n}\right)$ for all $n=0, \ldots, N$.

The basic idea is to discretize the Eq. (2) by using an implicit scheme for linear terms and an explicit scheme for the remaining terms. The goal is to reduce the execution time required to solve the equations by splitting up the terms in such a way that the stable time step for the explicit discretization is significantly smaller than the largest stable time step for the semi-implicit one.

After discretizing (2) with a semi-implicit first order scheme, we should find $u^{n+1}$ satisfying:

$$
\left\{\begin{array}{l}
\frac{u^{n+1}-u^{n}}{\tau}-\operatorname{div}\left(g\left(\left|\nabla u^{n}\right|\right) \nabla u^{n+1}\right)+\partial I_{K}\left(u^{n+1}\right) \ni 0 \\
\nabla u^{n+1} \cdot \vec{n}=0 \\
u^{n}=f
\end{array}\right.
$$
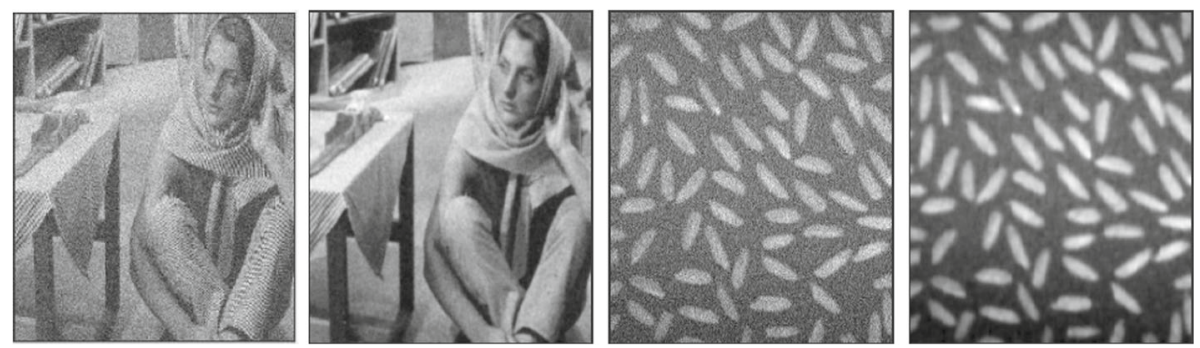

Fig. 5 Left to right: noisy Barbara image, restored Barbara image by our algorithm, noisy rice image, restored rice image by our algorithm 

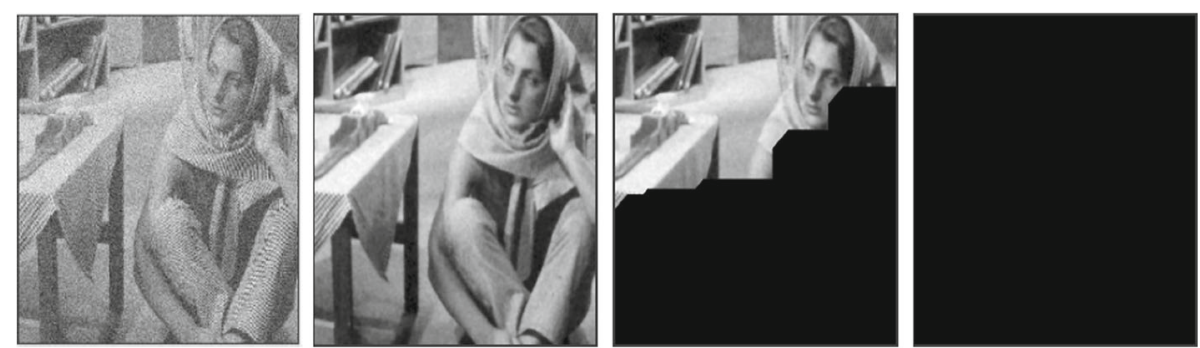

Fig. 6 Left to right: noisy Barbara image, restored image by PMPL with $p=12.5,16.5$, and 18.5, respectively

The corresponding discretization of the time splitting scheme (3)-(4) consists on finding at each time step, $v^{n+\frac{1}{2}}$ satisfying:

$$
\left\{\begin{array}{l}
\frac{v^{n+\frac{1}{2}}-v^{n}}{\tau}-\operatorname{div}\left(g\left(\left|\nabla v^{n}\right|\right) \nabla v^{n+\frac{1}{2}}\right)=0, \\
\nabla v^{n+\frac{1}{2}} \cdot \vec{n}=0, \\
v^{n}=f
\end{array}\right.
$$

followed by finding $w^{n+1}$ satisfying:

$$
\left\{\begin{array}{l}
\frac{w^{n+1}-w^{n+\frac{1}{2}}}{\tau}+\partial I_{K}\left(w^{n+1}\right) \ni 0 \\
\nabla w^{n+1} \cdot \vec{n}=0 \\
w^{n+\frac{1}{2}}=v^{n+\frac{1}{2}}
\end{array}\right.
$$

The Perona-Malik operator has been solved by different numerical methods and techniques and methods. To improve computational efficiency, we discretize (3) by (6) and we use the additive operator splitting scheme in the numerical implementation. On the other hand, the subdifferential Eq. (4), discretized by (7), is formulated as a minimization that will be solved by the dual formulation [25]. In the sequel, the two numerical methods are presented.

\subsection{Perona-Malik operator}

The simplest discretization of the $m$-dimensional Eq. (6) with reflecting boundary conditions is given by:

$$
\frac{u_{i}^{n+\frac{1}{2}}-u_{i}^{n}}{\tau}=\sum_{l=1}^{m} \sum_{j \in \mathcal{N}_{l}(i)} \frac{g_{i}^{n}+g_{j}^{n}}{2 . h^{2}}\left(u_{j}^{n+\frac{1}{2}}-u_{i}^{n+\frac{1}{2}}\right) \text {, }
$$

where pixel $i$ represents some location $x_{i}, u_{i}^{n}$ and $g_{i}^{n}$ denote, respectively, the approximation of $u\left(x_{i}, t_{n}\right)$ and $g\left(\left|\nabla u\left(x_{i}, t_{n}\right)\right|\right), m$ is the dimension size and $\mathcal{N}_{l}(i)$ consists of the two neighbors of pixel $i$ along the $l$ direction for all $l=1, \ldots, m$. In vector-matrix, notation (8) becomes:

$$
\frac{u^{n+\frac{1}{2}}-u^{n}}{\tau}=\sum_{l=1}^{m} A_{l}\left(u^{n}\right) u^{n+\frac{1}{2}} .
$$

The system matrix $A_{l}\left(u^{n}\right)$ is defined as $A_{l}\left(u^{n}\right)=\left[a_{\mathcal{I}, \mathcal{J}}^{l, n}\right]$, where

$$
a_{\mathcal{I}, \mathcal{J}}^{l, n}= \begin{cases}\sum_{\mathcal{J} \in \mathcal{N}_{l}^{-}(\mathcal{I})}^{-g_{\mathcal{J} \sim \mathcal{I}}^{n}} g_{\mathcal{J} \sim \mathcal{I}}^{n} & {\left[\mathcal{J} \in \mathcal{N}_{l}(\mathcal{I})\right],} \\ \mathcal{J} \in \mathcal{N}_{l}^{+}(\mathcal{I}) & \\ 0 & \text { (otherwise). }\end{cases}
$$

Therefore, we have:

$$
u^{n+\frac{1}{2}}=\left(I-\tau \cdot \sum_{l=1}^{m} A_{l}\left(u^{n}\right)\right)^{-1} u^{n} .
$$

The modification applied to (9) has been introduced firstly by [24] named as additive operator splitting (AOS) scheme which leads us to:

$$
u^{n+\frac{1}{2}}=\frac{1}{m} \sum_{l=1}^{m}\left(I-\tau \cdot m \cdot A_{l}\left(u^{n}\right)\right)^{-1} u^{n} .
$$

\subsection{The sub-differential flow}

The discretization of the problem (7) can be written as:

$$
w^{n+1}+\partial I_{K}\left(w^{n+1}\right) \ni w^{n+\frac{1}{2}} \text { for } n=0, \ldots, N .
$$

\begin{tabular}{|c|c|c|c|c|c|c|c|c|}
\hline \multirow[t]{2}{*}{ Images } & \multicolumn{2}{|l|}{ a } & \multicolumn{2}{|l|}{$b$} & \multicolumn{2}{|l|}{ c } & \multicolumn{2}{|l|}{$d$} \\
\hline & PSNR & SNR & PSNR & SNR & PSNR & SNR & PSNR & SNR \\
\hline Noisy & 20.1753 & 8.6159 & 20.2006 & 7.8502 & 20.1582 & 9.8390 & 20.1338 & 2.0217 \\
\hline Restored & 26.7594 & 15.2001 & 23.1807 & 10.8304 & 22.8471 & 12.5279 & 26.2332 & 8.1222 \\
\hline
\end{tabular}

where $\partial f$ denotes the sub-differential of a given function $f$.

Thanks to [25], we focus our attention on the projection $w^{n+1}=P_{K}\left(w^{n+\frac{1}{2}}\right)$. Indeed,

$$
J\left(w^{n+1}\right)=\frac{1}{2}\left\|w^{n+1}-w^{n+\frac{1}{2}}\right\|_{L^{2}(\Omega)}^{2}=\min _{z \in K} J(z),
$$

with $J(z)=\frac{1}{2}\left\|z-w^{n+\frac{1}{2}}\right\|_{L^{2}(\Omega)}^{2}$.

Table 1 PSNR and SNR values for the noisy and recovered images corresponding to the experiments shown in Figs. 2 and 3 

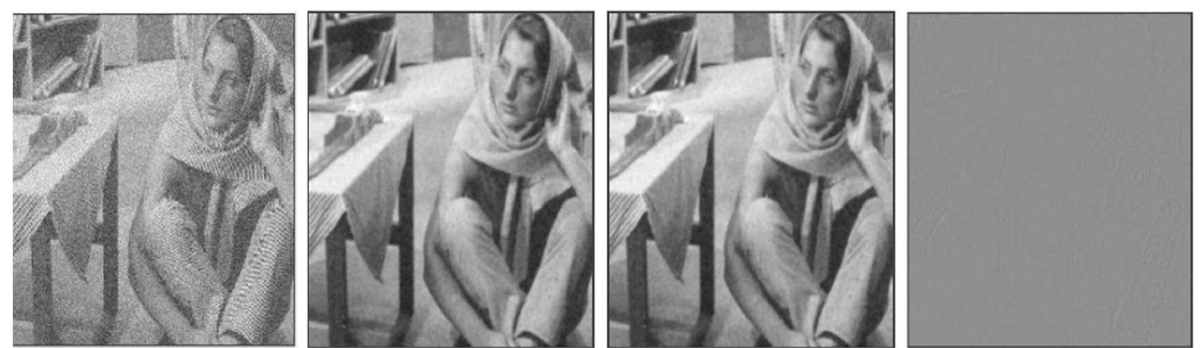

Fig. 7 Left to right: noisy image, restored Barbara image by our method, image obtained by PMPL model with $p=20$, the difference image

The dual formulation has been used to deal with this minimization problem. The dual problem associated with (12) is given by the following functional:

$G(z)=\int_{\Omega}(\operatorname{div}(z))^{2}+\int_{\Omega} w^{n+\frac{1}{2}} \operatorname{div}(z)+\lambda \int_{\Omega}|z|$.

In order to find the numerical solution of the functional (13), we denote by $z_{h}$ a minimizer of this functional and $G_{h}\left(z_{h}\right)$ the approximation of $G(z)$ (see [25] for instance):

$$
\begin{aligned}
G_{h}\left(z_{h}\right)= & \int_{\Omega}\left(\operatorname{div}\left(z_{h}\right)\right)^{2}+\int_{\Omega} w_{h}^{n+\frac{1}{2}} \operatorname{div}\left(z_{h}\right) \\
& +\lambda \sum_{\tau \in T_{h}}|\tau|\left|z_{h}\left(P_{\tau}\right)\right|,
\end{aligned}
$$

where $\tau$ represents simplex of the partitioning of $T_{h},|\tau|$ is the area of simplex $\tau$, and $P_{\tau}$ is one of the vertices of $\tau$. In image processing, $|\tau|$ could be seen as discretization step $h^{2}=1$.

In view of the fact that $G_{h}$ is nondifferentiable, we use a relaxation algorithm (see for instance [31] and the references therein) to minimize the functional (14) that can be summarized as follows:

1. Initiate the algorithm with vector $q^{0}$, set $k=0$, choose a canonical direction $e_{j} \in R^{n}$.

2. Solving the one-dimensional sub-problems $\min _{t \in R} \Psi_{j k}(t)$ where $\Psi_{j k}$ is defined as:

$$
\begin{aligned}
\Psi_{j k}: R & \rightarrow R \\
& t \rightarrow G_{h}\left(q^{k}+t e_{j}\right) .
\end{aligned}
$$

3. Taking $q^{k+1}=q^{k}+\omega t_{j k}$, where $\omega>0$ is an over-relaxation parameter.

4. We can use Newton algorithm to find $t_{j k}$, when $\Psi_{j k}$ is differentiable. Else, it can be computed directly.

5. The condition to stop this algorithm is $\left\|q^{k}-q^{k+1}\right\|_{l^{2}\left(R^{n}\right)} \leq \varepsilon$, for a given convergence tolerance $\varepsilon$.

At last, in the next section, results of numerical simulations are given.

\section{Numerical results and simulations}

This section is devoted to extensive numerical experimentations. The program will stop when it achieves our goal. Most algorithm parameters are chosen heuristically for the algorithms to perform their best. We set, the spatial size $h=1$ and the parameter $\omega=1,2$ of the relaxation algorithm. First, we illustrate the efficiency of our proposed numerical algorithm (cf. Figs. 2, 3, 4, and 5) for filtering the images corrupted with noise. As a second experiment, we will keep the same values of the previous parameters and we test the explicit scheme of the Perona-Malik and $p$-Laplacian (PMPL) proposed in [22] for different values of $p$ Fig. 6. We remark that the PMPL scheme depends on the parameter $p$ and for a large $p$, the restored image has been damaged. In order to avoid numerical instability, we must take a very small time step. So that, the needed simulation takes more time due to the nature of data. For that, in the third test, we evaluate the performance of our algorithm compared to PMPL scheme
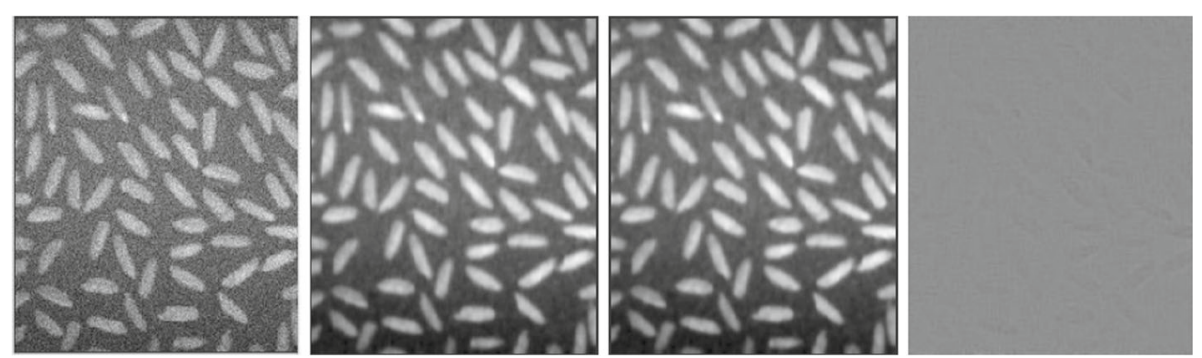

Fig. 8 Left to right: noisy image, restored rice image by our algorithm, image obtained by PMPL method with $p=20$, the difference image 
Table 2 CPU time of our algorithm and PMPL method for the Barbara and rice images

\begin{tabular}{lll}
\hline Images & Barbara & Rice \\
\hline Our method & $132.86 \times 10^{3} \mathrm{~ms}$ & $27.30 \times 10^{3} \mathrm{~ms}$ \\
Explicit schemes & $541.82 \times 10^{3} \mathrm{~ms}$ & $121.02 \times 10^{3} \mathrm{~ms}$ \\
\hline
\end{tabular}

by using CPU time. The last experiment aims to show the dependence also of the PMPL scheme to the threshold parameter $\lambda$.

For the improvements tests, we present the restorations and the results of our algorithm by choosing the parameter $\lambda=20$.

The restored images are clearly better than the noisy ones; to obtain an objective evaluation of the proposed method, the peak signal-to-noise ratio (PSNR) is used to measure the quality of the restoration results which is defined as:

$$
\operatorname{PSNR}=10 \log _{10}\left(\frac{255^{2} M N}{\left\|u_{0}-u\right\|_{2}^{2}}\right) d B
$$

where $u_{0}, u$, and $M \times N$ are the original image, the restored image and the size of the image, respectively. To qualify the restoration capacity of the method under consideration, the signal-to-noise ratio (SNR) is applied and denoted by:

$$
\mathrm{SNR}=\log _{10}\left(\frac{\sigma_{u}}{\sigma_{n}}\right) d B,
$$

where $\sigma_{u}$ and $\sigma_{n}$ are the signal and noise standard deviations, respectively. The value of these statistical measures indicates the strength of signal in restored images. Therefore, the value increases as the restored version of the image approaches the original one. In order to better evaluate the restoration process, PSNR and SNR values are shown in the Table 1.

Now, we are testing our method on Barbara and Rice images corrupted by Gaussian noise with zero mean and variance $\sigma^{2}=0.03$ and using the same parameters (Fig. 5).

The previous tests show the efficiency of our method. To argue the usage of the proposed algorithm, we will test, in the second experiment, the PMPL model using the explicit scheme proposed in [22] by using the same parameters of the previous test. We take different values of $p$ to study its impact on the image restoration.

Figure 6 shows that the PMPL method has restored the image, but when $p$ becomes large, the images are damaged due to the problem of stability which depends on the value of the parameters $p$ and $\lambda$ as well as the initial data. In order to repair the image and avoid numerical instability, we increase the value of the parameter $\lambda$ and decrease the time step size $d t$ until finding a suitable value for which the image will not get damaged. In this case, the PMPL scheme becomes stable for $d t=10^{-3}$ and $\lambda=60$. In Figs. 7 and 8, we will fix $\lambda=60$ and we present the comparison between our algorithm with $d t=0.1$ and the PMPL method with $d t=10^{-3}$ (Fig. 6).

The Figs. 7 and 8 show that the two methods give similar results. But in this experiment, our algorithm has restored the images in fewer time compared to the PMPL method (Table 2).

In the last test, we keep the same parameters employed in the previous experiment and change the value of the threshold $\lambda$ (Figs. 9 and 10).
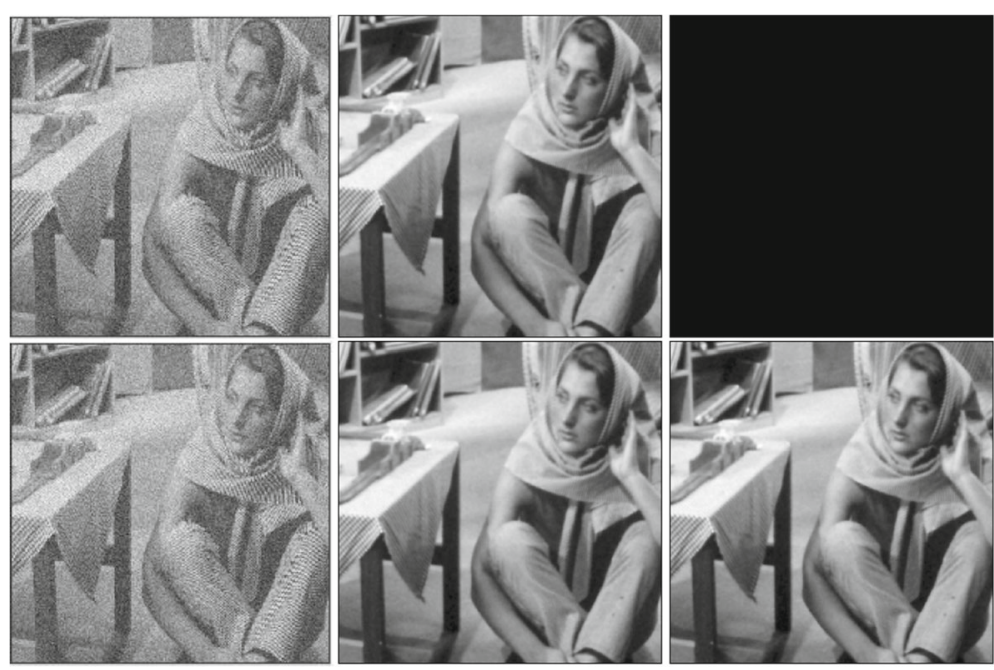

Fig. 9 Top row, restored Barbara image by PMPL method; bottom row, restored images by our algorithm; first column, noisy images; two right columns, recovered with $\lambda=40.98$ and $\lambda=40.97$, respectively 


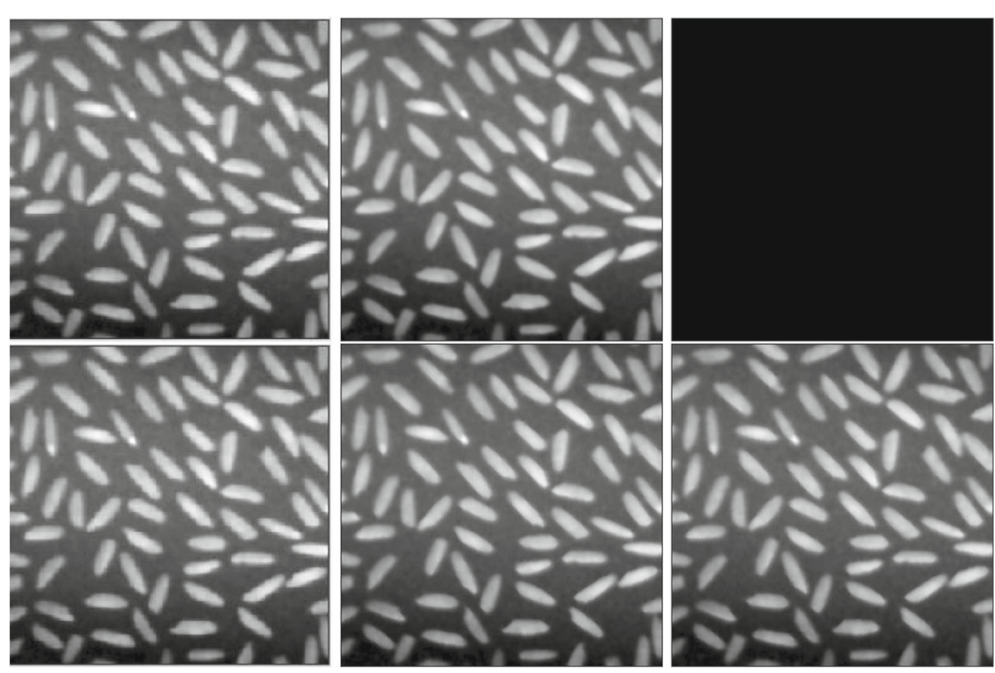

Fig. 10 Top row, restored Rice image by PMPL method; bottom row, restored images by our algorithm; first column, noisy images; two right columns, recovered with $\lambda=29.05$ and $\lambda=29$, respectively

In this experiment, the proposed algorithm proves to be performing better than the explicit scheme where the restored images are damaged when we took a $\lambda$ small and/or $p$ large (Fig. 9). The results of this section confirm the usefulness of our method that overcomes the condition of stability; whereas, we remark that for the explicit scheme, the stability criterion is given by $d t \leq f(p, \lambda)$ where $f$ is a decreasing function with respect to $p$ and a nondecreasing with respect to $\lambda$ such that

$$
f(p, \lambda) \longrightarrow 0 \text { as } p \rightarrow \infty \text { and } f(p, \lambda) \longrightarrow 0 \text { as } \lambda \rightarrow 0 .
$$

\section{Conclusions}

Perona and Malik proposed one of the pioneering model which represents an efficient and effective tool for image denoising. However, the numerical simulations produce a phenomenon known as the staircase effect, which causes images to look blocky. To overcome this, in [22], we have proposed a regularized model which is an interpolation of two classical models, Perona-Malik and $p$-Laplacian with $p \rightarrow \infty$. We have also demonstrate the efficiency and effectiveness of this model compared with the method most frequently used (see [22] for more detail). However, a major drawback for the numerical scheme is that the stability condition is strongly dependent to the parameters $p, \lambda$, and $d t$. For that, in this work, we develop a novel algorithm based on fractional step methods. Combining the classical additive operator splitting and a nonlinear relaxation algorithm, the numerical experiments demonstrate that the proposed algorithm is accurate and effective for images restoration. Comparing with the classical explicit schemes presented in [22] which strongly depends on the regularization methods and the model parameters, our algorithm controls the problem of stability and is significantly faster.

\section{Acknowledgements}

The authors gratefully acknowledge the helpful comments and suggestions of the reviewers, which have improved the presentation.

\section{Author's contributions}

All authors contributed equally to this work. All authors read and approved the final manuscript.

\section{Competing interests}

The authors declare that they have no competing interests.

\section{Publisher's Note}

Springer Nature remains neutral with regard to jurisdictional claims in published maps and institutional affiliations.

Received: 12 January 2017 Accepted: 29 May 2017

Published online: 24 June 2017

\section{References}

1. M Arian, N Manjari, B Richard, Anisotropic nonlocal means denoising. Appl. Comput. Harmon. Anal. 35, 452-482 (2013)

2. P Perona, J Malik, Scale-space and edge detection using anisotropic diffusion. IEEE Trans. Pattern Anal. Mach. Intell. 12(7), 629-639 (1990)

3. Y Wang, W Ren, $\mathrm{H}$ Wang, Anisotropic second and fourth order diffusion models based on convolutional virtual electric field for image denoising. Comput. Math. Appl. 66, 1729-1742 (2013)

4. Y Chen, S Levin, M Rao, Variable exponent, linear growth functionals in image restoration. SIAM J. Appl. Math. 66(4), 1383-1406 (2006)

5. R Aboulaich, D Meskine, A Souissi, New diffusion models in image processing. Comput. Math. Appl. 56(4), 874-882 (2008)

6. L Afraites, A Atlas, F Karami, D Meskine, Some class of parabolic systems applied to image processing. Discrete Contin. Dyn. Syst. Ser B 21. 6, 1671-1687 (2016)

7. P Guidotti, J Lambers, Two new nonlinear nonlocal diffusions for noise reduction. J. Math. Imaging Vis. 33(1), 25-37 (2009)

8. X Liu, L Huang, A new nonlocal total variation regularization algorithm for image denoising. Math. Comput. Simul. 97, 224-233 (2014)

9. L Rudin, S Osher, E Fatemi, Nonlinear total variation based noise removal algorithms. Physica D. 60, 259-268 (1992) 
10. O Seungmi, W Hyenkyun, Y Sangwoon, K Myungjoo, Non-convex hybrid total variation for image denoising. J. Vis. Commun. Image R. 24, 332-344 (2013)

11. L Shao, R Yan, $X L i, Y$ Liu, From heuristic optimization to dictionary learning: a review and comprehensive comparison of image denoising algorithms. IEEE Trans. Cybernet. 44, 177-189 (2014)

12. M Elad, On the origin of the bilateral filter and ways to improve it. IEEE Trans. Image Process. 11, 1141-1151 (2002)

13. R Yan, L Shao, L Liu, Y Liu, Natural image denoising using evolved local adaptive filters. Signal Process. 103, 36-44 (2014)

14. SG Chang, B Yu, M Vetterli, Adaptive wavelet thresholding for image denoising and compression. IEEE Trans. Image Process. 9, 1532-1546 (2000)

15. R Yan, L Shao, Y Liu, Nonlocal hierarchical dictionary learning using wavelets for image denoising. IEEE Trans. Image Process. 22, 4689-4698 (2013)

16. S Kichenassamy, The Perona-Malik paradox. SIAM J. Appl. Math. 75(5), 1328-1342 (1997)

17. F Catte, P Lions, JM Morel, T Coll, Image selective smooting and edge detection by nonlinear diffusion. SIAM J. Numer. Anal. 29(1), 182-193 (1992)

18. G Aubert, P Kornprobst, Mathematical problems in image processing. Partial differential equations and the calculus of variations. Second edition, vol. 147. (With a foreword by Olivier Faugeras. Applied Mathematical Sciences, 147. Springer, New York, 2006), p. xxxii+377

19. H Amann, Time-delayed Perona-Malik type problems. Acta Math. Univ. Comenian. 76(1), 15-38 (2007)

20. P Guidotti, A backward-forward regularization of the Perona-Malik equation. J. Diff. Equat. 252, 3226-3244 (2012)

21. P Guidotti, Y Kim, J Lambers, Image restoration with a new class of forward-backward-forward diffusion equations of Perona-Malik type with applications to satellite image enhancement. SIAM J. Imaging Sci. 6(3), 1416-1444 (2013)

22. A Atlas, F Karami, D Meskine, The Perona-Malik inequality and application to image denoising. Nonlinear Anal. Real World Appl. 18, 57-68 (2014)

23. RI McLachlan, G Reinout, W Quispel, Splitting methods. Acta Numerica. 11, 341-434 (2002)

24. J Weickert, BM ter Haar Romeny, MA Viergever, Efficient and reliable schemes for nonlinear diffusion filtering. IEEE Trans. Image Process. 7, 398-410 (1998)

25. S Dumont, N Igbida, On a dual formulation for the growing sandpile problem. Eur. J. Appl. Math. 2, 169-185 (2009)

26. E Dibendetto, Degenerate Parabolic Equations. (Spring-Verlag, New York, 1993)

27. W Wei, B Zhou, A p-Laplace equation model for image denoising. Inform. Technol. J. 11, 632-636 (2012)

28. F Andreu, JM Mazon, JD Rossi, J Toledo, The limit as $p \rightarrow \infty$ in a nonlocal $p$-Laplacian evolution equation: a nonlocal approximation of a model for sandpiles. Calc. Var. 3, 279-316 (2009)

29. L Prigozhin, Variational model of sandpile growth. Euro. J. Appl. Math. 3 225-235 (2009)

30. R Glowinski, J Rappaz, Approximation of a nonlinear elliptic problem arising in a non-Newtonian fluid model in glaciology. M2AN Math. Model. Numer. Anal. 1, 175-186 (2003)

31. N Igbida, F Karami, T Nga, Discrete collapsing sandpile model. Nonlinear Anal. Theory Methods Appl. 1, 177-189 (2014)

\section{Submit your manuscript to a SpringerOpen ${ }^{\circ}$ journal and benefit from:}

- Convenient online submission

- Rigorous peer review

- Open access: articles freely available online

- High visibility within the field

- Retaining the copyright to your article

Submit your next manuscript at $>$ springeropen.com 